Stratifying by stage the residual tumour $(\mathrm{OR}=2.4$; $\mathrm{p}=0.0001)$, age $(\mathrm{OR}=1.9 \mathrm{P}=0.0001)$, and the performance status $(\mathrm{OR}=1.2 ; \mathrm{p}=0.03)$ resulted as independent survival prognostic factors according to Cox multivariate analysis.

Conclusion* Our data suggest that patients aged $\geq 70$ can tolerate radical surgical treatments in the same way as younger patients without a significant increase in morbidity and, obviously, without ignoring the appropriate geriatric precautions. Furthermore, maximal surgical effort with optimal cytoreduction should be considered the gold standard regardless of age.

Therefore, our data underlines the importance of managing these patients within Gynecologic Oncology units equipped with a multidisciplinary team.

\section{OVARIAN CANCER AND BRCA1 AND 2 GERMLINE MUTATIONS - THE PORTUGUESE EXPERIENCE}

${ }^{1} \mathrm{M}$ Peixoto*, ${ }^{2} S$ Dâmaso, 1' Cunha Carvalho, ${ }^{2} \mathrm{R}$ Paiva, ${ }^{1} \mathrm{~V}$ Gonçalves, 'S Broco, ${ }^{1} \mathrm{~T}$ Carvalho, ${ }^{1} \mathrm{C}$ Pinto, ${ }^{1} \mathrm{I}$ Pazos, 'G Sousa. 'IPO Coimbra Francisco Gentil, Medical Oncology, Coimbra, Portugal; ${ }^{2}$ Hospital Santa Maria, Medical Oncology, Lisbon, Portugal

\subsection{6/ijgc-2021-ESG0.458}

Introduction/Background* Ovarian cancer (OC) is the second most common gynecologic malignancy in developed countries and the third most common in developing countries. Approximately 13 to 15 percent of OC are attributable to heritable mutations in BRCA1 and 2.

This study aims to: I- assess median overall survival (mOS); II- characterize patients (pts) with OC assessed in a Family Risk Consultation (FRC).

Methodology This is a multicentric, descriptive and retrospective study of pts with OC followed at the FRC between 2007-2019 in two porruguese hospitals. Data was obtained from clinical files. Statistical analysis was performed using SPSS version $24^{\oplus}$ and OS using Kaplan-Meier method.

Result(s)* There were included 70 pts, of which 23\% $(n=16)$ had BRCA1/2 mutation: BRCA1 mutation occurred in $56 \%$ $(\mathrm{n}=9)$ of pts and BRCA2 in $44 \% \quad(\mathrm{n}=7)$. The Portuguese founder mutation - BRCA2 c.156-157insAlu was found in 2 pts.

Median age of BRCA mutated (mut) pts was 56 years (3971) and BRCA wild type (wt) was 62 years (35-78).

The most frequent histology was Serous Carcinoma, in 86\% ( $\mathrm{n}=60$ ) of pts; most frequent stages were IIIC 46\% (n $=32)$ and IV $17 \%(\mathrm{n}=12)$. Neoadjuvant chemotherapy (CT) was performed in 50\% of pts $(\mathrm{n}=35)$ and in $37 \%$ ( = 26) surgery was the first therapeutic approach followed by adjuvant CT. Eleven pts (16\%) were treated with PARP inhibitors: 6 pts BRCAmut and 5 BRCAwt.

There was family history of cancer in 56\% of BRCAmut and in $45 \%$ of BRCAwt.

mOS of BRCAmut was 13.81 years (CI 95\% 10.36-17.26) and 5.54 years (CI 95\% 4.21-6.88) to BRCAwt, with a significant difference between the two groups $\left(X^{2}=4.460\right.$; $\mathrm{P}=0.035)$.

Conclusion* Detection rate of BRCA1/2 mut was higher than described in literature. BRCAmut pts showed a statistically significant longer survival, when compared with BRCA wt pts. Characterization of these pts at a national level would be an opportunity to obtain real data from the Portuguese population.

\section{3} OUR EXPERIENCE IN OVARIAN CANCER 2006-2015 . STANDARDS OF QUALITY IN SURGICAL MANAGEMENT

0 Arencibia Sanchez*, AF Rave Ramirez, D González García-Cano, M Laseca Modrego, A Martín Martínez. Complejo Hospitalario Universitario Insular Materno Infantil de Gran Canaria, Gynecologic Oncology, las palmas de gran canaria, Spain

\subsection{6/ijgc-2021-ESGO.459}

Introduction/Background* It is important to know the survival data of patients with ovarian cancer treated in our unit, the variables associated with the prognosis and the degree of compliance with the standards in the surgical management of ovarian cancer

Methodology Retrospective study of patients with ovarian cancer diagnosed and treated in CHUIMI in the period between 2006-2015. We studied epidemiological variables,stage at diagnosis,type of treatment,histopathological study,follow up and current status of patients.

Result(s)* The total number of patients diagnosed with ovarian cancer in the study period was 331, with a mean age of 57.84 years (range 26-85 years).69.8\% were in advanced stages at the time of diagnosis (Stage I 23.9\% (79),Stage II 6.3\% (21), Stage III 54.1\% (179) and Stage IV 15.7\% (52).

Regarding the histological type,serous was the most frequent representing $49.8 \%$ of the sample, followed by endometrioid with $16.3 \%$ and clear cells with $10.9 \%$. We found that endometrioid,clear cells and mucinous types were more frequent in the grupo diagnosed with eraly stages versus the serous type that were more associated with the advanced disease.

Overall survival (OS) at 5 years is $40.8 \%$ for the complete series. $83.3 \%$ for stages I, $72.2 \%$ for stages II, $29.1 \%$ for stages III and there are no patients in stage IV who lived after 5 years. In stages III, the most frequent therapeutic approach is initial surgery in $41.1 \%$, followed by neoadjuvant chemotherapy in $30.3 \%$. Stage III patients receiving surgery + adjuvant chemotherapy showed an OS of $47 \%$ at 5 years (median survival 44 months) meanwhile those who received neoadyuvant chemotherapy and get the surgery in second place showed an OS of $27.1 \%$ (median survival 35 months).

When we studied the effect of tumor residue after surgery in stage III patients, the OS when the surgery was complete was $52,9 \%$ at five years, $15 \%$ if there were residual tumour, regardless of the size. Initial surgery was performed in 58\% of all stages III-IV (objective> 50\%). Complete citoreduction was achieved in $51 \%$ of all stages III-IV (minimum objective> $50 \%$, optimal > 65\%)

Conclusion* Our epidemiological and survival data coincide with what has been published in the literature.Having surgeons with experience in the management of peritoneal carcinomatosis will allow to increase the rate of complete citoreductions

\section{ESGO CERTIFICATION FOR ADVANCED OVARIAN CANCER SURGERY: THE EXPERIENCE OF AN ONCOLOGY CENTER TO AIM ACCREDITATION}

${ }^{1} V$ Bernardini*, ${ }^{2} S$ Giulia, ${ }^{1} D V$ Antonella, ${ }^{1} G$ Stefano, ${ }^{1} \mathrm{C}$ Giuseppa, ${ }^{2} \mathrm{G}$ Marina, ${ }^{1} \mathrm{~K}$ Sami, ${ }^{1} \mathrm{P}$ llaria, 'A Grazia, ${ }^{1} \mathrm{~B}$ Enrico. ${ }^{1}$ Cà Foncello, Treviso, Italy; ${ }^{2}$ Ospedale Padova, Padova, Italy

10.1136/ijgc-2021-ESGO.460

Introduction/Background* The outcomes of advanced ovarian cancer surgery is related to the size of the largest residual 